\title{
Criteria for Modification of Ventilator Settings in Critically ill Children: A Pilot Study
}

\author{
Allen Eddington ${ }^{1}$, Guillaume Emeriaud ${ }^{1}$, Philippe Jouvet ${ }^{1}$, LChristopher Newth ${ }^{2}$, Dominik Novotni ${ }^{3}$ and \\ Marc Wysocki ${ }^{4}$
}

${ }^{1}$ Pediatric ICU, Sainte-Justine Hospital, Montreal, Canada,

${ }^{2}$ Children's Hospital Los Angeles, USA

${ }^{3}$ Hamilton Medical, Bonaduz, Switzerland,

${ }^{4}$ Research Center, Sainte Justine Hospital, Canada

Received: February 17, 2018; Published: February 27, 2018

*Corresponding author: Philippe Jouvet, Pediatric ICU, Sainte-Justine Hospital, Montreal, Canada, Tel: 1514345 4927;

Email: philippe.jouvet@umontreal.ca

\section{Abstract}

Aim: Patient care protocols and mechanical ventilator modes capable of auto-adjusting settings based on integrated, non invasive monitoring techniques are being developed to improve the quality of paediatric mechanical ventilation. We performed a study to describe which criteria intensivists currently use to make ventilator setting changes.

Methods: Critically ill children admitted to the intensive care units at Sainte-Justine Hospital (SJH) and the Children's Hospital Los Angeles (CHLA) was included throughout all phases of invasive mechanical ventilation. The reasons for ventilator setting modifications were recorded by caregivers in real time. Temporary modifications made during suctioning or subject manipulations were excluded.

Results: Twenty subjects were included at Sainte-Justine Hospital and fifteen at the Children's Hospital Los Angeles. The mean duration of electronic capture of ventilator setting modifications was around 6 days in both centers. Excluding changes to $\mathrm{FiO}_{2}$, the median number of setting changes per subject per day was 2.5 at $\mathrm{HSJ}$ and 0.9 at $\mathrm{CHLA} \mathrm{PaCO}_{2}$ was identified as the main primary reason for respiratory rate, tidal volume or positive aspiratory pressure changes at $\mathrm{SJH}$ and $\mathrm{pH}$ was the main primary reason at $\mathrm{CHLA}_{\text {. EtCO }}$ was not used frequently as the primary reason for adjustments in both hospitals. Pulse oximetry was also identified as the main primary reason for $34.1 \%$ at $\mathrm{SJH}$ and $25 \%$ at CHLA of changes to PEEP. Except for $\mathrm{FiO}_{2}$, less than the half of the changes of ventilator settings were based on elements which were potentially in corpora table into automatic protocols.

Conclusion: This study reveals that physician over-estimate the role of blood $\mathrm{pH}$ and $\mathrm{PCO}_{2}$ in their ventilation management strategies. Furthermore, roughly half the changes to PEEP, $40 \%$ of the changes to respiratory rate, tidal volume or positive aspiratory pressure could potentially be managed by automatic ventilator modes based on technology which already exists.

Keywords: Mechanical Ventilation; Automated Ventilation; Paediatric Intensive Care

Abbrevations: CHLA: Children’s Hospital Los Angeles; SJH: Sainte-Justine Hospital; IRB: Institutional Review Board; PICU: Pediatric Intensive Care Units; PIP:Positive Aspiratory Pressure; PS: Pressure Support; PEEP: Positive End-Expiratory Pressure

\section{Introduction}

Invasive mechanical ventilation in children is managed by attending physicians, fellows, residents, nurses and respiratory therapists [1]. However, little evidence is available to describe how these caregivers manage invasive mechanical ventilation on a dayto-day basis. Caregivers have multiple variables to consider when implementing or adjusting a ventilation strategy, including at the least the patient's age, weight, chronic illness, acute illness, level of sedation, physical examination and chest radiography findings, blood gas analysis and noninvasive monitoring. Once a ventilation strategy is chosen and a mode of ventilation and settings are prescribed, changes in any of these factors can potentially lead to changes in the settings, the mode, or even the overall strategy. It has been established in recent point prevalence studies that significant variability exists in the settings used including settings outside of current recommendations for specific diseases [2-5].

It is unclear whether this practice variation can be explained by specific patient condition or is simply explained by practice heterogeneity. If the latter is true, this suggests that a more uniform 
best practice - potentially leading to improved patient outcome has yet to be described, accepted and implemented. This practice variability is also a barrier to the evaluation of different ventilation strategies and new modes of ventilation, because there is no clearly described "best practice" to use as a standard of comparison. It may also contribute to significant background patient-to-patient variation among patients enrolled in studies not directly related to ventilation management, and this variation may mask the effect (positive or negative) of those studies' interventions [6]. The multiplicity of factors influencing pediatric ventilation and the wide variations in current practice make imparting expertise to medical trainees a challenge.

Furthermore, computer assisted ventilation management protocols and automatic modes of ventilation modes in which continuously monitored data is relayed directly to the ventilator which then adjusts the settings are currently being developed [7-11]. Caregivers are unlikely to trust or use these protocols or automatic modes of ventilation if their algorithms cannot be demonstrated to lead to ventilator management comparable or superior to current practice. Such a comparison cannot be made without first describing the current practice [12]. The present pilot study in two centers identified the key elements among the multiplicity of invasive and non-invasive monitored data at caregivers' disposition and describes the relationships between these elements and specific ventilator setting changes.

\section{Subjects and Methods}

This is a prospective observational study carried out in two centers between January 2010 and January 2011 at the pediatric intensive care units (PICU) of Sainte-Justine hospital (SJH) and between November 2011 and February 2012 at the PICU of the Children's Hospital Los Angeles (CHLA), two tertiary care pediatric hospitals. The Institutional Review Board (IRB) of both institutions approved this study.

\section{Subjects}

During the study periods, all consecutive critically ill children were considered eligible to participate in the study if they met the following inclusion criteria:

A. Presence of invasive mechanical ventilation (via end tracheal tube or tracheotomy) and

B. Expected duration of mechanical ventilation more than 3 days.

The latter was determined by the presence of one of the following 3 criteria that were demonstrated as risk factors of prolonged mechanical ventilation ${ }^{12}$ :

a) mean airway pressure $\geq 13 \mathrm{cmH}_{2} \mathrm{O}$ maintained for at least 60 minutes at any time in the first 24 hours of mechanical ventilation,

b) PRISM $\geq 10$ on the day of PICU admission, or

c) Continuous infusion of a sedating medicine for any amount of time during the first 24 hours of mechanical ventilation.

Subjects were excluded if they met any of the following criteria:

a) Presence of a "Do not resuscitate" or "Do not reinitiate" order in the chart,

b) Suspected or confirmed brain death,

c) History of mechanical ventilation (invasive or non invasive) at home, or

d) The data gathering computer was not available.

\section{Identification of the Factors that Influence Ventilator Setting Changes}

In order to generate necessary data to prepare for this study, we performed two surveys amongst the paediatric intensivists, neonatologists, paediatric intensive care fellows, and neonatology fellows of SJH. In the first survey, participants were asked to identify the elements they considered when increasing or decreasing the imposed respiratory rate, positive aspiratory pressure or tidal volume, positive end-expiratory pressure, and fraction of inspired oxygen. All questions were open-ended. The survey was distributed via e-mail and in paper format to twenty individuals, and eight surveys were completed (forty percent response rate). The results of the first survey were reviewed, and similar responses were combined. The second survey included the elements identified in the first survey, and asked respondents to estimate the frequency (in terms of a percentage) with which a particular element was included in the decision-making process amongst all of their prescriptions to change a particular ventilator setting (e.g., Among all of your prescriptions to change the imposed respiratory rate, what percentage are based on the partial pressure of carbon dioxide from an arterial blood gas?).

The format required that participants write a percentage for each of seventeen questions. Again, the survey was distributed to twenty individuals and thirteen individuals responded (sixtyfive percent response rates). The results of the first survey were instrumental in the design of a computer program used as the primary data gathering utility for this project (see below). The results of the second survey-specifically, that seventy-eight percent of changes to the imposed rate, positive aspiratory pressure, set tidal volume, and pressure support are made in reference to arterial blood $\mathrm{pH}$ or arterial partial pressure or carbon dioxide and that eighty-one percent of changes to the fraction inspired oxygen and positive end-expiratory pressure are made in reference to pulse oximetry monitoring-served as the basis for the hypothesis of this study.

\section{Study Design}

When a subject was selected for the study (see above), a laptop computer with a custom-designed computer program (Figure 1) was installed at the bedside. The computer was used to record each ventilator setting change along with one primary reason for the ventilator setting change and an unlimited number of secondary reasons. The caregiver changing the setting filled out this electronic 
form at the bedside and the time the change was made, from prepopulated scrolling lists of setting changes, primary and secondary reasons. Manual entries were however also allowed if changes and reasons to change were not found on the lists. The role of the caregiver responsible for the setting change (nurse, respiratory therapist, resident, fellow or attending) was also recorded for each change.

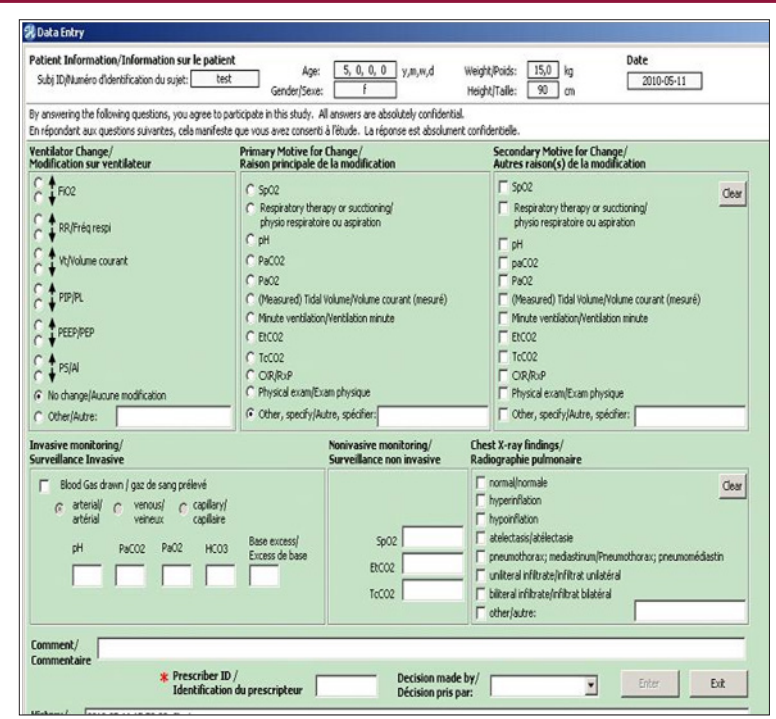

Figure 1: Screen shot of the data-gathering software

\section{Sample Size}

Based on an average prevalence of motivating factors for ventilator change equal to $50 \%$ (P0) and a 95\% confidence interval, measurement of 384 ventilator changes were required to reach a level of precision of $+/-5 \%$ around the estimate. Also, based on an expected mean length of mechanical ventilation of 3 days and an average of 7 measurements per day, a sample-size of 20 subjects per center (approx. 420 measurements per center) was targeted.

\section{Data Collection}

Subject characteristics including demographic data, diagnosis, severity scores, clinical data at inclusion and outcomes were collected from the charts. The endpoint of this study was the change to the ventilator setting in reference to the primary and secondary motivating factors. Specifically, for the purpose of this study, ventilator changes were classified as the following: change of mode of ventilation; increase or decrease by any amount of mechanically imposed respiratory rate, aspiratory volume, peak aspiratory pressure, positive end-expiratory pressure, pressure support, or fraction of inspired oxygen. Anticipated motivating factors include the following: pulse oximetry, $\mathrm{pH}$, partial pressure of carbon dioxide, partial pressure of oxygen, tidal volume, minute ventilation, end-tidal carbon dioxide, chest radiograph, session of chest physical therapy or respiratory toilet and the physical examination.

In addition to these anticipated motivating factors, other motivating factors could be identified and recorded in the course of the study. We tracked the following changes: Rate (RR), Tidal Volume (Vt), Positive Aspiratory Pressure (PIP), Pressure Support (PS), Positive End-Expiratory Pressure (PEEP), and Fraction of Inspired Oxygen $\left(\mathrm{FiO}_{2}\right)$. Monitored elements (any finding, whether clinical, laboratory, radiological, or derived from measurements from the ventilator) were identified if they played a role in the decisionmaking process leading to a ventilator setting change. However, no interpretation of findings or values was given. Therefore, the relationship between monitored elements and ventilator setting changes are expressed as the percentage of ventilator setting changes for which a particular monitored element played a role. Caregiver compliance with the protocol was estimated by recording the internal ventilator log of ventilator setting modifications using a compact flash reader connected to the ventilator (Servo-I, Marquette, Solana, Sweden).

The ventilator setting changes recorded by the ventilator log were then matched with the ventilator setting changes recorded by caregivers with a custom-designed computer program (Figure 1). The number of setting changes in the ventilator log with a corresponding entry in the study software was divided by the total number of setting changes in the ventilator log to yield a percent compliance. The compliance was measured in $10 \%$ of the subjects at Sainte-Justine Hospital. Between the SJH and CHLA studies, we modified the methodology in order to focus the data collection on ventilation parameter changes and to decrease study workload [1]. We no longer included $\mathrm{FiO}_{2}$ setting changes; [2]. The internal log of the ventilator was no longer recorded. In order to estimate the potential gains of employing either computer assisted ventilation management protocols or ventilator modes with automatic setting adjustment, we categorised the reasoning for any given setting change as readily able to be incorporated into an automated protocol based on current technology, or not able to be assimilated.

The reasons we considered able to be embodied included spontaneous respiratory rate, measured positive aspiratory 
pressure, measured tidal volume, minute ventilation, oxygen pulse oximetry, end tidal $\mathrm{PCO}_{2}$, planned stepwise weaning. The reasons not able to be incorporated into an automated protocol included blood gases $\left(\mathrm{pH}, \mathrm{PaCO}_{2}, \mathrm{PcCO}_{2}, \mathrm{PaO}_{2}\right)$, physical examination and chest film findings. When multiple elements were reported for one ventilator setting change, if any one element was considered not readily able to be incorporated into an automatic protocol, that ventilator setting change was considered to be based on nonincorporated elements.

\section{Statistical Analysis}

Data are described as mean and SD, unless otherwise specified, for quantitative variables and frequencies for qualitative variables ( $\mathrm{n}=$ number of ventilator setting changes). Prevalence of motivating factors for ventilator setting modifications is described as percentage. Motivating factors are classified as primary and secondary criteria.

Table 1: Characteristics of the children included in the study. Data are expressed as mean \pm standard deviation or percentage. HSJ: Sainte-Justine Hospital, CHLA: Children's Hospital Los Angeles PRISM: Pediatric risk of mortality score20, PELOD score: pediatric logistic organ dysfunction score21, PICU LOS: length of stay in the pediatric intensive care unit, EtCO2: end tidal PCO2.

\begin{tabular}{|c|c|c|}
\hline \multirow{2}{*}{ Characteristics } & \multicolumn{2}{|c|}{ Subjects } \\
\cline { 2 - 3 } & SJH & CHLA \\
\cline { 2 - 3 } & $\mathbf{( n = 2 0 )}$ & $6.6 \pm 6.3$ \\
\hline Age (years old) & $2.4 \pm 2$ & $28.8 \pm 27.2$ \\
\hline Weight (kg) & $12.1 \pm 7.1$ & 31 \\
\hline Female gender (\%) & 50 & $7.8 \pm \pm 6.5$ \\
\hline PRISM & $10 \pm 6$ & $15.9 \pm 8.3$ \\
\hline PELOD score at inclusion & $8 \pm 6$ & $21.0 \pm 13.4$ \\
\hline PICU LOS (d) & $29 \pm 69$ & $16.8 \pm 12$ \\
\hline Duration of ventilation (d) & $28 \pm 70$ & 6.3 \\
\hline 28-day mortality (\%) & 15.0 & $5.7 \pm 2.6$ \\
\hline Duration of electronic capture (d) & $6 \pm 10$ & 100 \\
\hline EtCO2 monitoring (\%) & 35 & \\
\hline
\end{tabular}

Table 2: Number of ventilation settings adjustements recorded during the study period at Sainte-Justine Hospital (HSJ) and Children's Hospital of Los Angeles (CHLA). FiO2 adjustments are excluded. RR: respiratory rate, PIP: positive inspiratory pressure (including pressure support level), Vt: tidal volume, PEEP: positive end expiratory pressure.

\begin{tabular}{|c|c|c|c|c|}
\hline & RR adjustments & PIP or Vt adjustments & PEEP adjustments & Total adjustments \\
\hline SJHn (\%) & $60(28)$ & $111(53)$ & $37(18)$ & 208 \\
\hline CHLA $\mathrm{n}(\%)$ & $33(42)$ & $37(47)$ & $8(10)$ & 78 \\
\hline Total $\mathrm{n}(\%)$ & $93(32)$ & $148(51)$ & $45(16)$ & 286 \\
\hline
\end{tabular}

The frequency with which respondents identified secondary monitored elements, assuming a primary element was identified, varied as follows: RR $20 \%$ and $44 \%$, Vt-PIP $19.3 \%$ and 50\%, PEEP $17.1 \%$ and $37.5 \%, \mathrm{FiO}_{2} 2.7 \%$ and not available, at HSJ and CHLA respectively. The median caregiver compliance with the protocol was $74.5 \%$ at HSJ. No trends in compliance were found between the different shifts or over the days of observation for each subject. For the different ventilator settings, there were between $3.4 \%$ and $16.1 \%$ of changes for which respondents did not record their motivation for making the change. $\mathrm{PaCO}_{2}$ was identified as the main primary reason for RR, Vt or PIP changes at HSJ and $\mathrm{pH}$ was the main primary reason at CHLA. $\mathrm{EtCO}_{2}$ was not used frequently as the primary reason for RR, Vt or PIP adjustments in both hospitals (Tables 3 \& 4).

Pulse oximetry was also identified as the main primary reason for $34.1 \%$ at HSJ and $25 \%$ at CHLA of changes to PEEP, followed by physical exam, $\mathrm{PaO}_{2}$, chest radiography findings, planned step-wise weaning and measured tidal volume (Tables 3 \& 4). After exclusion of temporary changes made during interventions (end tracheal tube aspiration and other subject manipulation), pulse oximetry 
was identified as the primary monitored element for $84.5 \%$ of $\mathrm{FiO}_{2}$ changes (Table 3). Except for $\mathrm{FiO}_{2}$, less than the half of the

changes of ventilator settings were based on elements which were potentially in corpora table into automatic protocols (Table 5).

Table 3: Primary clinical or laboratory parameters that motivated a change in a dedicated ventilator setting at Sainte-Justine hospital, on a total of 905 settings change.

\begin{tabular}{|c|c|c|c|c|}
\hline & $\mathbf{R R}$ & Vt or PIP & PEEP & FiO2 \\
\hline & $n=60$ & $\mathrm{n}=111$ & $\mathbf{n}=\mathbf{3 7}$ & $\mathrm{n}=697$ \\
\hline \multicolumn{5}{|c|}{ Ventilator parameters } \\
\hline $\begin{array}{c}\text { Abnormal spontaneous RR } \\
(\%)\end{array}$ & 1.2 & 5.4 & 0 & 0 \\
\hline Abnormal measured Vt (\%) & 0 & 18.9 & 7.3 & 0.1 \\
\hline $\begin{array}{c}\text { Abnormal measured PIP } \\
(\%)\end{array}$ & 0 & 1.8 & 0 & 0 \\
\hline $\begin{array}{l}\text { Abnormal minute } \\
\text { ventilation }(\%)\end{array}$ & 0 & 1.8 & 0 & 0 \\
\hline \multicolumn{5}{|c|}{ Non invasive monitoring } \\
\hline Pulse oximetry (\%) & 2.5 & 4.5 & 34.1 & 26.3 \\
\hline EtCO2 (\%) & 1.2 & 0.9 & 0 & 0 \\
\hline \multicolumn{5}{|c|}{ Blood gases parameters motivating changes } \\
\hline pH (\%) & 8.6 & 4.5 & 2.4 & 0 \\
\hline PCO2 (\%) & 42 & 20.7 & 0 & 0 \\
\hline $\mathrm{PaO2}(\%)$ & 0 & 0.9 & 9.8 & 0.3 \\
\hline \multicolumn{5}{|c|}{ Others } \\
\hline Physical Exam (\%) & 4.9 & 3.6 & 12.2 & 0.3 \\
\hline CXR findings (\%) & 0 & 0 & 9.8 & 0 \\
\hline Weaning protocol (\%) & 3.8 & 5.4 & 7.3 & 0 \\
\hline Interventions* (\%) & 12.3 & 21.6 & 0 & 68.9 \\
\hline Miscellaneous (\%) & 23.5 & 9.9 & 17.1 & 4.1 \\
\hline Total (100) & 100 & 100 & 100 & 100 \\
\hline
\end{tabular}

Table 4: Primary clinical or laboratory parameters that motivated a change in a dedicated ventilator setting in 15 children at the

Children Hospital Los Angeles.

\begin{tabular}{|c|c|c|c|}
\hline & RR & Vt or PIP & PEEP \\
\hline & $\mathrm{n}=33$ & $\mathrm{n}=\mathbf{3 7}$ & $n=8$ \\
\hline \multicolumn{4}{|c|}{ Ventilator parameters } \\
\hline Abnormal spontaneous RR (\%) & 0 & 0 & 0 \\
\hline Abnormal measured Vt (\%) & 3 & 10.8 & 0 \\
\hline Abnormal measured PIP (\%) & 0 & 0 & 0 \\
\hline Abnormal minute ventilation (\%) & 0 & 5.4 & 0 \\
\hline \multicolumn{4}{|l|}{ Non invasive monitoring } \\
\hline Pulse oximetry (\%) & 3 & 2.7 & 25 \\
\hline EtCO2 (\%) & 12.1 & 5.4 & 0 \\
\hline \multicolumn{4}{|c|}{ Blood gases parameters motivating changes } \\
\hline pH (\%) & 51.5 & 45.9 & 0 \\
\hline PCO2 (\%) & 6.1 & 10.8 & 0 \\
\hline $\mathrm{PaO} 2(\%)$ & 0 & 0 & 37.5 \\
\hline \multicolumn{4}{|l|}{ Others } \\
\hline Physical Exam (\%) & 0 & 0 & 0 \\
\hline CXR findings (\%) & 3 & 5.4 & 0 \\
\hline
\end{tabular}




\begin{tabular}{|c|c|c|c|}
\hline Weaning protocol (\%) & 3 & 2.7 & 0 \\
\hline Miscellaneous (\%) & 18.2 & 10.8 & 37.5 \\
\hline Total (\%) & 100 & 100 & 100 \\
\hline
\end{tabular}

Table 5: Reasons to modify ventilator settings that are currently incoporatable into an automatic ventilator mode. RR: respiratory rate, Vt: tidal volume, PIP: positive inspiratory pressure, PEEP: positive end expiratory pressure and FiO2, HSJ: Sainte-Justine Hospital, CHLA: Children's Hospital Los Angeles.

\begin{tabular}{|c|c|c|}
\hline \multirow{2}{*}{} & \multicolumn{2}{|c|}{$\begin{array}{c}\text { Percentage of changes incorporatable currently } \\
\text { into an automated ventilator mode }\end{array}$} \\
\hline Ventilator parameter(s) modified & HSJ & 15 \\
\hline RR (\%) & 33 & 35 \\
\hline Vt or PIP (\%) & 51 & 14 \\
\hline PEEP (\%) & 51 & - \\
\hline FiO2 (\%) & 97 & \\
\hline
\end{tabular}

\section{Discussion}

Contrary to the results of a survey in which caregivers estimated that roughly eighty percent of their prescribed setting changes to the set respiratory rate, positive aspiratory pressure, tidal volume and pressure support were motivated by $\mathrm{PaCO}_{2}$ or blood $\mathrm{pH}$, those elements were cited as primary elements in a third of setting changes when caregivers recorded their reasoning at the time the prescription. At CHLA, this rate increased to one half suggesting that ventilator settings adjustments also depend on the organizational structure of each PICU. The rate of modification of ventilator settings in children is below 3 times a day when $\mathrm{FiO}_{2}$ changes are excluded. As expected, $\mathrm{FiO}_{2}$ settings are modified essentially on pulse oximetry values.

As one would expect, by selecting incubated subjects with a projected duration of mechanical ventilation of greater than 3 days, the subjects included in this study had longer lengths of stay, higher PRISM and PELOD scores, and a higher mortality rate than the average for our ICU. The number of ventilator settings changes, excluding $\mathrm{FiO}_{2}$, was not greatly different in the two centers studied (median of 2.5 and 0.9 changes per subject and per day). This is much lower than the rate at which an automatic protocol usually performs (more than 1 change per hour) [7,13,14]. Reasons for modification of $\mathrm{FiO}_{2}$ are consistent with perceived clinical experience, as demonstrated by the fact that the routine management of the $\mathrm{FiO}_{2}$ has been largely delegated to bedside nurses, who are instructed to adjust $\mathrm{FiO}_{2}$ in order to maintain target pulse oximetry readings and in accordance with the patient's skin coloration and general status.

Our findings show that $97.1 \%$ of $\mathrm{FiO}_{2}$ setting changes in our center could potentially be managed by automatically adjusting ventilator modes. Such technology is currently available for children above $7 \mathrm{~kg} 13$, and is also of particular interest in neonatal intensive care units $[15,16]$. Modifications to PEEP were made with reference to several elements; however, multiple elements were only recorded for $17.1 \%$ of modifications. In other words, there are several elements to track when managing PEEP, but usually, only one of these elements becomes clinically significant at a time. Pulse oximetry was by far the most frequently cited element (about one third of the changes), while physical exam, $\mathrm{PaO} 2$, chest radiography findings, planned step-wise weaning and measured tidal volume were all identified at similar low frequencies. Assuming that an adaptable weaning plan can be incorporated into computer assisted decision-making protocols and/or automatically adjusting ventilator modes; about half of the changes to PEEP which we observed could have been managed by such methods [13]. Of note, heart-lung interactions were never specifically reported as a reason to modify the level of PEEP.

This could be explained first by assuming that when physicians cited the physical exam as a reason for changing PEEP, heart-lung interactions were considered as part of that assessment [17]. And second, by recalling that we observed changes only to established ventilator settings as opposed to initial settings and/or magnitude of change. Therefore, patients whose initial assessment included cardiovascular reasons to limit PEEP may have had their PEEP modified as a function of pulse oximetry and the other elements mentioned above, but with smaller increments of change and lower total values throughout their entire course of ventilation than those without special heart-lung considerations. It is also possible that heart-lung interactions were considered in decisions of not modifying the PEEP, which were not recorded in this study. Another interesting finding was that no method for setting PEEP based on an assessment point of derecruitment (pressure-volume curve, compliance change, volumetric capnography, esophageal pressure) were used in PICUs. Esophageal pressure is probably rarely used in pediatric patients.

According to our results, modifying PEEP and $\mathrm{FiO}_{2}$ according to $\mathrm{SpO}_{2}$ is not the current clinical practice although it is recommended in the literature, at least in ARDS patients $[5,13,18,19]$. One of the most interesting findings in our study was the relationship between blood $\mathrm{pH}$ or $\mathrm{PCO}_{2}$ level versus RR, PIP, Vt and PS. PCO2 level or blood $\mathrm{pH}$ was cited as the primary element in only one third of these setting changes at SJH. This refutes a common perception among physicians as indicated by a local survey of Ste-Justine Hospital practitioners who reported that blood $\mathrm{pH}$ or $\mathrm{PCO}_{2}$ was the 
primary reason (80\%) for the change in RR, PIP, Vt and PS (data not shown). That would imply that most changes to those settings were made either because the $\mathrm{PCO}_{2}$ or $\mathrm{pH}$ was outside an acceptable target range or because targets were met, and physicians felt that the patient could be weaned without those elements going out of target range.

But according to our study observations, this was actually practiced in less than half of the setting changes made. Rather, other considerations caused caregivers to modify ventilator settings, namely peak pressures, measured tidal volumes, spontaneous respiratory rates, etc being out of target range.

When considering secondary elements, and categorising the monitored elements as able or not to be incorporated into automatic ventilator modes, less than a half of the setting changes to RR, PIP Vt and PS could have been managed by use of automatic ventilator modes using readily available technology. Of note, $\mathrm{EtCO}_{2}$ monitoring is not usual practice in HSJ PICU (7/20 subjects had $\mathrm{EtCO}_{2}$ monitored) but it was usual practice at Children's Hospital Los Angeles (100\% had $\mathrm{EtCO}_{2}$ monitored). However less than $15 \%$ of ventilator settings where triggered by the $\mathrm{EtCO}_{2}$, suggesting that this parameter is not crucial currently for ventilator setting prescription $[20,21]$.

Caution should be used when interpreting our data because it represents the practice of two centers in an area of medicine with few standards or guidelines. The results may not be generalizable to patients who are rapidly weaned after PICU admission. In addition, we identified only those monitored elements that led to ventilator changes. If the default position of a ventilation management plan was to change (wean) a setting, and a monitored parameter prevented that change, we did not capture such information. Also, as is the case with any study attempting to understand clinical reasoning, our observations are limited to what clinicians reported, and may therefore be inherently somewhat oversimplified.

\section{Conclusion}

This pilot study demonstrates that observation and surveying of clinical decision making in mechanical ventilation at the time decisions are being made is feasible and that the results may lead to a more accurate and objective understanding of our own practise. Specifically, these initial results reveal that physician over-estimate the role of blood $\mathrm{pH}$ and $\mathrm{PCO}_{2}$ in their ventilation management strategies. Furthermore, roughly half the changes to PEEP, $40 \%$ of the changes to RR, Vt, PIP, and PS, and $97 \%$ of changes to FiO2 in one of the two centers could potentially be managed by automatic ventilator modes based on technology which already exists. As non invasive means of tracking $\mathrm{PCO}_{2}$ become more sophisticated, or with the integration of intermittent blood gas results into automatic ventilator modes or computer assisted decision making protocols (by manual entry and validation or through automatic entry from the hospital's laboratory system and manual result validation) greater strides can be made in the standardisation of mechanical ventilation.
Such advances would allow for large scale studies to determine best practices and would allow patients with simpler courses of mechanical ventilation to be managed through use of protocols allowing paediatric intensivists use their time and efforts on more complex management problems. In order for such advances to take place, physicians must become comfortable with the capabilities and limitations of automatic modes of ventilation as well as with the technology that feeds information into such systems.

\section{References}

1. Newth C, Jouvet P (2015) Organization Characteristics in North America. In: Rimensberger PC, editor. Pediatric and Neonatal Mechanical Ventilation. Springer Berlin Heidelberg, Berlin, Germany, pp. 1571-1574.

2. Santschi M, Jouvet P, Leclerc F, Gauvin F, Newth CJ, et al. (2010) Acute lung injury in children: therapeutic practice and feasibility of international clinical trials. Pediatr Crit Care Med 11(6): 681-689.

3. Santschi M, Gauvin F, Hatzakis G, Lacroix J, Jouvet P, et al. (2007) Acceptable respiratory physiologic limits for children during weaning from mechanical ventilation. Intens Care Med 33(2): 319-325.

4. Santschi M, Randolph AG, Rimensberger PC, Jouvet P (2013) Mechanical ventilation strategies in children with acute lung injury: a survey on stated practice pattern*. Pediatr Crit Care Med 14(7): e332-e337.

5. Khemani RG, Sward K, Morris A, Dean JM, Newth CJ, et al. (2011) Variability in usual care mechanical ventilation for pediatric acute lung injury: the potential benefit of a lung protective computer protocol. Intens Care Med 37(11): 1840-1848.

6. Morris AH (2000) Developing and implementing computerized protocols for standardization of clinical decisions. Ann Intern Med 132(5): 373-383.

7. Jouvet PA, Payen V, Gauvin F, Emeriaud G, Lacroix J (2013) Weaning children from mechanical ventilation with a computer-driven protocol: a pilot trial. Intens Care Med 39(5): 919-925.

8. Wysocki M, Jouvet P, Jaber S (2014) Closed loop mechanical ventilation. Journal of clinical monitoring and computing 28(1): 49-56.

9. Burns KE, Lellouche F, Nisenbaum R, Lessard MR, Friedrich JO (2014) Automated weaning and SBT systems versus non-automated weaning strategies for weaning time in invasively ventilated critically ill adults. The Cochrane database of systematic reviews 9: CD008638.

10.10. Rose L, Schultz MJ, Cardwell CR, Jouvet P, McAuley DF, et al. (2013) Automated versus non-automated weaning for reducing the duration of mechanical ventilation for critically ill adults and children. The Cochrane database of systematic reviews 6(6): CD009235.

11. Arnal JM, Wysocki M, Novotni D, Demory D, Lopez R, et al. (2012) Safety and efficacy of a fully closed-loop control ventilation (IntelliVent-ASV(R)) in sedated ICU patients with acute respiratory failure: a prospective randomized crossover study. Intens Care Med 38(5): 781-787.

12. Payen V, Jouvet P, Lacroix J, Ducruet T, Gauvin F (2012) Risk factors associated with increased length of mechanical ventilation in children. Pediatr Crit Care Med 13(2): 152-157.

13. Jouvet P, Eddington A, Payen V, Bordessoule A, Emeriaud G, et al. (2012) A pilot prospective study on closed loop controlled ventilation and oxygenation in ventilated children during the weaning phase. Crit Care 16(3): R85.

14. Jouvet P, Farges C, Hatzakis G, Monir A, Lesage F, et al. (2007) Weaning children from mechanical ventilation with a computer-driven system (closed-loop protocol): a pilot study. Pediatr Crit Care Med 8(5): 425432 .

15. Claure N, Bancalari E (2013) Automated closed loop control of inspired oxygen concentration. Respiratory care 58(1): 151-161. 
16. Hallenberger A, Poets CF, Horn W, Seyfang A, Urschitz MS, et al. ( 2014) Closed-loop automatic oxygen control (CLAC) in preterm infants: a randomized controlled trial. Pediatrics 133(2): e379-e385.

17. Ingaramo OA, Ngo T, Khemani RG, Newth CJ (2014) Impact of positive end-expiratory pressure on cardiac index measured by ultrasound cardiac output monitor*. Pediatr Crit Care Med 15(1): 15-20.

18. N Engl J Med (2000) Ventilation with lower tidal volumes as compared with traditional tidal volumes for acute lung injury and the acute respiratory distress syndrome. The Acute Respiratory Distress Syndrome Network 342(18):1301-1308.

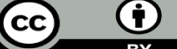

This work is licensed under Creative Commons Attribution 4.0 License

Submission Link: http://biomedres.us/submit-manuscript.php
19. (2015) Pediatric acute respiratory distress syndrome: consensus recommendations from the Pediatric Acute Lung Injury Consensus Conference 16(5): 428-439.

20. Pollack MM, Ruttimann UE, Getson PR (1988) Pediatric risk of mortality (PRISM) score. Crit Care Med 16(11): 1110-1116.

21. Leteurtre S, Martinot A, Duhamel A, Proulx F, Grandbastien B, et al. (2003) Validation of the paediatric logistic organ dysfunction (PELOD) score: prospective, observational, multicentre study. Lancet 362(9379): 192-197.

$\begin{array}{ll}\text { BIOMEDICAL } & \text { Assets of Publishing with us } \\ \text { RESEARCHES } & \text { - Global archiving of articles } \\ \text { - Immediate, unrestricted online access }\end{array}$

